\title{
Porcine Extrahepatic Vascular Endothelial Asialoglycoprotein Receptor 1 Mediates Xenogeneic Platelet Phagocytosis In Vitro and in Human-to-Pig Ex Vivo Xenoperfusion
}

Anjan K. Bongoni, ${ }^{1,2,3}$ David Kiermeir, ${ }^{3}$ Julie Denoyelle, ${ }^{1,3}$ Hansjörg Jenni, ${ }^{4}$ Christopher Burlak, ${ }^{5}$ Jörg D. Seebach, ${ }^{6}$ Esther Vögelin, ${ }^{3}$ Mihai A. Constantinescu, ${ }^{3}$ and Robert Rieben ${ }^{1,3}$

Background. Asialoglycoprotein receptor-1 (ASGR1) mediates capture and phagocytosis of platelets in pig-to-primate liver xenotransplantation. However, thrombocytopenia is also observed in xenotransplantation or xenoperfusion of other porcine organs than liver. We therefore assessed ASGR1 expression as well as ASGR1-mediated xenogeneic platelet phagocytosis in vitro and ex vivo on porcine aortic, femoral arterial, and liver sinusoidal endothelial cells (PAEC/PFAEC/PLSEC). Methods. Porcine forelimbs were perfused with whole, heparinized human or autologous pig blood. Platelets were counted at regular intervals. Pig limb muscle and liver, as well as PAEC/PFAEC/PLSEC, were characterized for ASGR1 expression. In vitro, PAEC cultured on microcarrier beads and incubated with non-anticoagulated human blood were used to study binding of human platelets and platelet-white blood cell aggregation. Carboxyfluorescein diacetate succinimidyl ester-labeled human platelets were exposed to PAEC/PFAEC/PLSEC and analyzed for ASGR1-mediated phagocytosis. Results. Human platelet numbers decreased from $102 \pm 33$ at beginning to $13 \pm 6 \times 10^{3} / \mu \mathrm{L}(P<0.0001)$ after 10 minutes of perfusion, whereas no significant decrease of platelets was seen during autologous perfusions (171 \pm 26 to $\left.122 \pm 95 \times 10^{3} / \mu \mathrm{L}\right)$. The PAEC, PFAEC, and PLSEC all showed similar ASGR1 expression. In vitro, no correlation was found between reduction in platelet count and platelet-white blood cell aggregation. Phagocytosis of human carboxyfluorescein diacetate succinimidyl ester-labeled platelets by PAEC/PFAEC/PLSEC peaked at 15 minutes and was inhibited $(P<0.05$ to $P<0.0001)$ by rabbit anti-ASGR1 antibody and asialofetuin. Conclusions. The ASGR1 expressed on aortic and limb arterial pig vascular endothelium plays a role in binding and phagocytosis of human platelets. Therefore, ASGR1 may represent a novel therapeutic target to overcome thrombocytopenia associated with vascularized pig-toprimate xenotransplantation.

(Transplantation 2015;99: 693-701)

(1)

enotransplantation of porcine organs and cells may help to expand the pool of available donor organs for clinical transplantation. However, despite considerable progress over the past decades, immune responses and other barriers prevent clinical application. The use of organs stemming

Received 27 May 2014. Revision requested 10 June 2014.

Accepted 30 September 2014.

'Department of Clinical Research, University of Bern, Bern, Switzerland.

2 Graduate School for Cellular and Biomedical Sciences, University of Bern, Bern, Switzerland.

${ }^{3}$ Clinic of Plastic and Hand Surgery, University Hospital, Bern, Switzerland.

${ }^{4}$ Clinic of Cardiovascular Surgery, University Hospital, Bern, Switzerland.

${ }^{5}$ Department of Surgery, Schulze Diabetes Institute, University of Minnesota, Minneapolis, MN.

${ }^{6}$ Division of Immunology and Allergology, University Hospital and Medical Faculty, Geneva, Switzerland.

This study was supported by the Swiss National Science Foundation (32003B_135272, 32003B_138434, and 320030-138376), and the German Research Foundation (Transregio CRC 127).

The authors declare no conflicts of interest.

A.K.B. participated in the research design, writing of the article, performance of the experiments, data analysis. D.K. participated in performing the animal experiments from genetically modified pigs has prevented hyperacute rejection, antibody-mediated or cellular acute rejection, at least in animal models. ${ }^{1-3}$ Nevertheless, other issues related to physiological and immunological incompatibilities, including thrombotic microangiopathy and systemic consumptive coagulopathy, need to be solved. ${ }^{4-6}$ The latter are characterized by platelet activation, aggregation, and sequestration leading

and the overall design of the study. J.D. participated in performing laboratory analyses. H.J. participated in performing the animal experiments and the overall design of the study. C.B. and J.D.S. provided scientific support and reagents and participated in the critical revision of the article. E.V. and M.A.C. participated in the concept and design of the study and carried part of the responsibility. R.R. participated in the concept and design of the study, analyzing the data, writing the article, and carried the main responsibility for the study.

Correspondence: Robert Rieben, PhD, University of Bern, Department of Clinical Research, Murtenstrasse 50, P.O. Box 44, CH-3010 Bern, Switzerland. (robert.rieben@dkf.unibe.ch).

Supplemental digital content (SDC) is available for this article. Direct URL citations appear in the printed text, and links to the digital files are provided in the HTML text of this article on the journal's Web site (www.transplantjournal.com).

Copyright ( 2015 Wolters Kluwer Health, Inc. All rights reserved.

ISSN: 0041-1337/15/9904-693

DOl: $10.1097 / T P .0000000000000553$ 
A

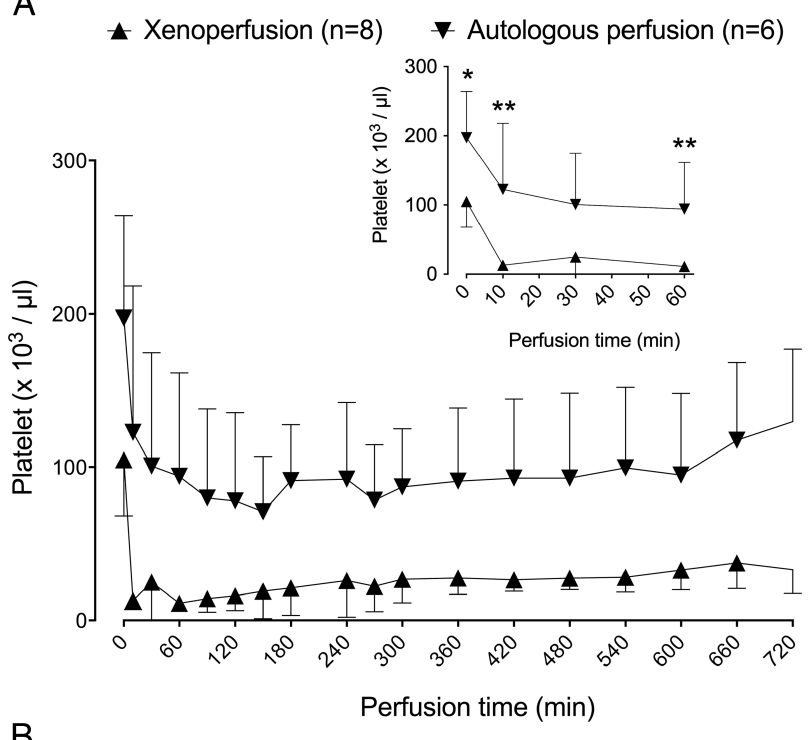

B

\begin{tabular}{|l|l|l|}
\hline $\begin{array}{l}\text { Unpaired t-Test (area under the curve over the } \\
\text { whole perfusion period) }\end{array}$ & Significant? & $\begin{array}{l}\text { Two-tailed } \\
\text { p value }\end{array}$ \\
\hline Xenoperfusion vs. Autologous perfusion & $\star \star$ & 0.002 \\
\hline
\end{tabular}

FIGURE 1. Platelet counts during ex vivo perfusion of porcine forelimbs with heparinized human or autologous pig blood. Forelimbs amputated from large white pigs of both sexes, weighing 30 to $40 \mathrm{~kg}$, were connected to an extracorporeal perfusion circuit, and then xenogeneic or autologous perfusions were performed. Perfusate blood samples were collected at regular time intervals and platelets counted using a Sysmex analyzer. A, A rapid loss of platelet counts was observed in xenogeneic, but not in autologous perfusions. The inset shows the enlarged graph for the first 60 minutes of perfusion with indication of significant differences between autologous versus xenoperfusion (one-way ANOVA with Bonferroni correction for individual time points; ${ }^{\star} P \leq 0.05,{ }^{\star *} P \leq 0.01$ ). Xenogeneic perfusions (xeno) $(n=8)$ and autologous perfusions (auto) $(n=6)$. B, Statistical analysis was carried out for area under the curve of the whole 720 minutes perfusions using the unpaired 2-tailed Student $t$ test $\left.{ }^{* \star} P \leq 0.005\right)$. ANOVA, analysis of variance.

to a dysregulation of the coagulation system and eventually to organ dysfunction.

Thrombocytopenia has been observed previously in pig-to-primate xenotransplantation models, especially in pig-to-baboon liver transplantation ${ }^{8}$ and during ex vivo porcine liver perfusion with human blood. ${ }^{9}$ Although initial hepatic function, including coagulation, was proven to be satisfactory, the development of thrombocytopenia is currently precluding pig liver xenotransplantation, even as a "bridge" to allotransplantation. ${ }^{10}$ Platelet activation and aggregation are critical events that might precede the development of thrombocytopenia and/or sequestration of platelets in xenografts. ${ }^{11}$ However, platelet phagocytosis by endothelial cells through receptor-ligand interactions cannot be excluded. Recently, it has been shown that porcine Kupffer cells and liver sinusoidal endothelial cells (LSEC) bind and phagocytose human platelets in ex vivo perfusion systems. ${ }^{12}$ Many scavenger receptors have been reported on LSEC that facilitate the clearance of particulate, molecular, and cellular "waste" from the circulation, ${ }^{13}$ one of them being the asialoglycoprotein receptor-1 (ASGR1). It functions as a recycling receptor, which mediates capture and phagocytosis of Gal $\beta 1-4-$ and GalNAcß1-4-terminating glycoproteins. ${ }^{14,15}$ Other functions of ASGR1 that have been put forward include clearance of asialoglycoproteins ${ }^{16}$ and apoptotic cells. ${ }^{17}$ Asialoglycoprotein receptor-1 may also participate in cell-cell, cell-matrix, or intramatrix interactions. ${ }^{18}$ The receptor is expressed on porcine Kupffer cells, hepatocytes ${ }^{19}$ and on porcine LSEC (PLSEC). Finally, ASGR1 on PLSEC mediates phagocytosis of human platelets. ${ }^{20}$ Asialoglycoprotein receptor-1-mediated human platelet binding depends on the availability of carbohydrate binding sites on ASGR1 as well as exposed carbohydrate ligands on platelets, such as terminal Gal $\beta 1-4$ and GalNAcß $1-4$ residues. Recently, it has been shown that, compared to porcine platelets, human platelets have 4 times more Gal $\beta 1-4 \mathrm{GlcNAc}$ and GlcNAc $31-4 G l c N A c$ ligands for ASGR1. ${ }^{21}$

In summary, thrombocytopenia after liver xenotransplantation and ex vivo xenoperfusion is a well-recognized phenomenon. In contrast, little is known on platelet phagocytosis mediated by other endothelial sites. Here, we report that ex vivo xenoperfusion of amputated porcine forelimbs with human blood using extracorporeal perfusion ${ }^{22}$ leads to an immediate loss of human platelets from circulation. We therefore examined ASGR1 expression on porcine vascular endothelial cells (EC) including aortic EC (PAEC), femoral arterial (PFAEC), and PLSEC. In addition, the role of ASGR1 for binding and phagocytosis of human platelets was tested in vitro.

\section{MATERIALS AND METHODS}

\section{Ex Vivo Perfusion Model}

Forelimbs of nontransgenic, large white pigs of both sexes, weighing 30 to $40 \mathrm{~kg}$, were used to perform ex vivo perfusion with $500 \mathrm{~mL}$ of whole, heparinized (Liquemin, 10,000 IU/ $500 \mathrm{~mL}$ ) anticoagulated human blood (xenoperfusion) or autologous blood (autologous perfusion), for up to 12 hours as described by Bongoni et al. ${ }^{22}$ Biopsies and blood samples were collected at regular time intervals and blood cell counts performed using an automated hematology analyzer (Sysmex KX21N; Sysmex Suisse AG, Horgen, Switzerland) to measure changes in platelet counts.

\section{Endothelial Cell Isolation and Culture}

Porcine vascular EC (PAEC and PFAEC) were isolated using a previously described method. ${ }^{23}$ The PLSEC isolation from porcine liver was performed according to a previously described method. ${ }^{20,21}$

\section{Platelet Isolation and Carboxyfluorescein Diacetate Succinimidyl Ester Labeling}

Freshly isolated human platelets were labeled with carboxyfluorescein diacetate succinimidyl ester (CFSE) using CellTrace CFSE Cell Proliferation Kit (Life Technologies) according to manufacturer's protocols.

\section{Immunofluorescence}

Porcine ECs on 8-well Lab-Tek chamber slides (Milian Suisse SA, Vernier, Switzerland) and slides with $5 \mu \mathrm{m}$ cryosections of porcine limb muscle were fixed in $3.7 \%$ formaldehyde or acetone, respectively, for 5 minutes and then incubated with endothelial specific antibodies: rat anti-pig CD31 (R\&D Systems, Abingdon, United Kingdom), goat antihuman VE-cadherin (cross-reacting with porcine VEcadherin, Santa Cruz Biotechnologies Inc., Dallas, TX), rabbit antihuman ASGR1 (cross-reacting with porcine ASGR1; ${ }^{20}$ GenWay Biotech, San Diego, CA), and mouse antihuman 
CD41 (FITC-labeled; Dako Schweiz AG, Baar, Switzerland) for 2 hours at room temperature or overnight at $4^{\circ} \mathrm{C}$. Thereafter, slides were washed and exposed to fluorescence conjugated secondary antibodies: donkey anti-rat immunoglobulin (Ig) G Cy3 (Jackson Immunoresearch, West Grove, PA), donkey anti-goat IgG Alexa 488 (Molecular Probes, Leiden, The Netherlands), and sheep antirabbit IgG Cy3, (Sigma, St. Louis, MO). The slides were analyzed using a confocal laserscanning microscope (LSM710; Zeiss, Jena, Germany).

\section{In Vitro Human Blood Clotting Assay}

The PAEC were cultured on collagen-coated Biosilon microcarrier beads as described previously by Kohler et al and Wuensch et al. ${ }^{24,25}$ Beads with confluent PAEC were incubated with non-anticoagulated whole human blood. For more details, see SDC, http://links.lww.com/TP/B100).

\section{In Vitro Platelet Phagocytosis-Cell Enzyme-Linked Immunosorbent Assay}

Analysis of human platelet phagocytosis by porcine vascular EC was performed based on the assay described by Paris et al. ${ }^{20}$ In brief, $4 \times 10^{7}$ CFSE-labeled human platelets were added to wells on 96-well plates containing confluent EC. For inhibition assay, EC were preincubated with polyclonal rabbit anti-ASGR1 antibody, polyclonal rabbit Ig G as isotype control (Sigma) or asialofetuin (Sigma) for 30 minutes before addition of human platelets. The fluorescence of CFSE was acquired at $492 \mathrm{~nm}$ (reference wavelength $517 \mathrm{~nm}$ ) using a Tecan Infinite M1000 microplate reader.

\section{In Vitro Platelet Phagocytosis_Immunofluorescence Staining}

The CFSE-labeled human platelets, $4 \times 10^{7}$, were added to confluent EC on 8-well chamber slides for 60 minutes at $37^{\circ} \mathrm{C}$. After fixation, the slides were stained with mouse anti-pig CD107a (AbD Serotec, Kidlington, Oxford, United Kingdom), anti-CD31, anti-VE-cadherin, or anti-ASGR1 and appropriate fluorescence-labeled secondary antibodies. Slides were analyzed using a Zeiss LSM710 confocal laser-scanning microscope.

For further details in Materials and Methods, see SDC, http://links.lww.com/TP/B100.

\section{RESULTS}

\section{Ex Vivo Perfusion Model of Xenotransplantation-Induced Platelet Loss}

A rapid loss of circulating human platelets was observed during xenoperfusion of pig forelimbs with human blood, whereas in autologous pig blood perfusions only a slow decrease of platelet numbers was seen. The average platelet counts were $236.3 \pm 28.9 \times 10^{3} / \mu \mathrm{L}$ in human blood and $301 \pm 67.2 \times 10^{3} / \mu \mathrm{L}$ in pig blood. The counts dropped about 2 -fold to $102.2 \pm 32.9 \times 10^{3} / \mu \mathrm{L}$ and $171.3 \pm 26.4 \times 10^{3} / \mu \mathrm{L}$, respectively, after the first run through the perfusion system before getting into contact with porcine tissue. These reductions in platelet counts are explained by the dilution of the blood with hydroxyethyl starch solution (Voluven, Fresenius) which was used for priming of the extracorporeal circuit. After 10 minutes of xenoperfusion with human blood, platelet counts dropped approximately 10 -fold, from $102.2 \pm 32.9 \times 10^{3} / \mu \mathrm{L}$ at the beginning to $13.0 \pm 6.1 \times$ $10^{3} / \mu \mathrm{L}(P=0.048)$. In autologous pig blood perfusions, the respective values after 10 minutes were not significantly lower than at onset $\left(171.3 \pm 26.4 \times 10^{3} / \mu \mathrm{L}\right)$, namely, $122.7 \pm 95.5 \times$ $10^{3} / \mu \mathrm{L}$, but significantly $(P=0.009)$ higher than the ones of the respective xenoperfusions. After 60 minutes of perfusion, platelet counts were $11.3 \pm 4.1 \times 10^{3} / \mu \mathrm{L}$ in xenoperfusions and $94.0 \pm 67.4 \times 10^{3} / \mu \mathrm{L}$ in autologous perfusions, respectively, and stayed at these levels throughout the remaining perfusion time (720 minutes in total, Figure 1). Platelet counts, measured as area under the curve over the whole perfusion period, were significantly lower in xenogeneic perfusions $(P=0.002, t$ test $)$ as compared to autologous perfusions.

\section{ASGR1 Expression by Porcine Vascular \\ Endothelial Cells}

As shown by immunofluorescence and confocal microscopy, cultured PAEC, PFAEC, and PLSEC were positive for CD31 and ASGR1 (Figure 2A) as well as positive for VE-cadherin and ASGR1 (Figure S1A, SDC, http://links.lww.com/TP/B100). Fluorescence intensity of ASGR1 staining was quantitated using ImageJ software (NIH), and significance was tested using 1-way analysis of variance with Bonferroni correction. No difference in ASGR1 expression was observed between the different types of EC tested $(P>0.72$; Figure S1B, SDC, http://links.lww.com/TP/B100). Expression of ASGR1 on porcine vascular EC was further confirmed using flow cytometry. PAEC, PFAEC, and PLSEC were CD31/ASGR1 double positive for $91.5 \%, 95.1 \%$, and $94.9 \%$ of the total cell numbers, with no difference in ASGR1 expression levels (Figure 2B to D). The respective controls were also tested and are represented in Figure 2E to G. Western blot analysis of whole cell lysates using PAEC, PFAEC, and PLSEC all showed ASGR1 protein expression as a band at $\sim 50 \mathrm{kDa}$ (Figure $2 \mathrm{H}$ ), with no significant differences in expression levels as analyzed by ImageJ software (Figure 2I).

\section{ASGR1 Protein Expression and Platelet Deposition on Pig Limb Tissue Samples}

Porcine limb muscle (Figure 3A to B) and liver (Figure 3C to D) tissue samples were all positive for ASGR1 expression as assessed by immunofluorescence. The fact that staining for VE-cadherin and CD31, respectively, colocalized with ASGR1 in all biopsy sections suggests that ASGR1 is indeed expressed on endothelial cells, corresponding to the observations made in vitro using cultured porcine vascular EC. However, quantitative analysis showed significantly higher $(P<0.0001)$ ASGR1 staining on liver tissue as compared to limb, supposedly due to the known expression of ASGR1 on hepatocytes (Figure 3E).

Muscle tissue biopsies from xenoperfusions were also analyzed for human platelet infiltration and sequestration by staining for CD41, a human platelet marker. As shown in Figures 3F to $G$, a higher number of human platelets was found in the muscle tissue after 1 hour of perfusion $(48 \pm 11$ platelets per high-power viewing field $)$ as compared with 12 hours of perfusion $(1 \pm 2, P<0.0001)$. Furthermore, fluorescence microscopy analysis of tissue samples after 1 hour of perfusion showed that sequestered platelets (CD41, green) were colocalized (yellow) with CD31 (Figure 3H, white arrows) as well as ASGR1 (red, Figure 3I, white 
A
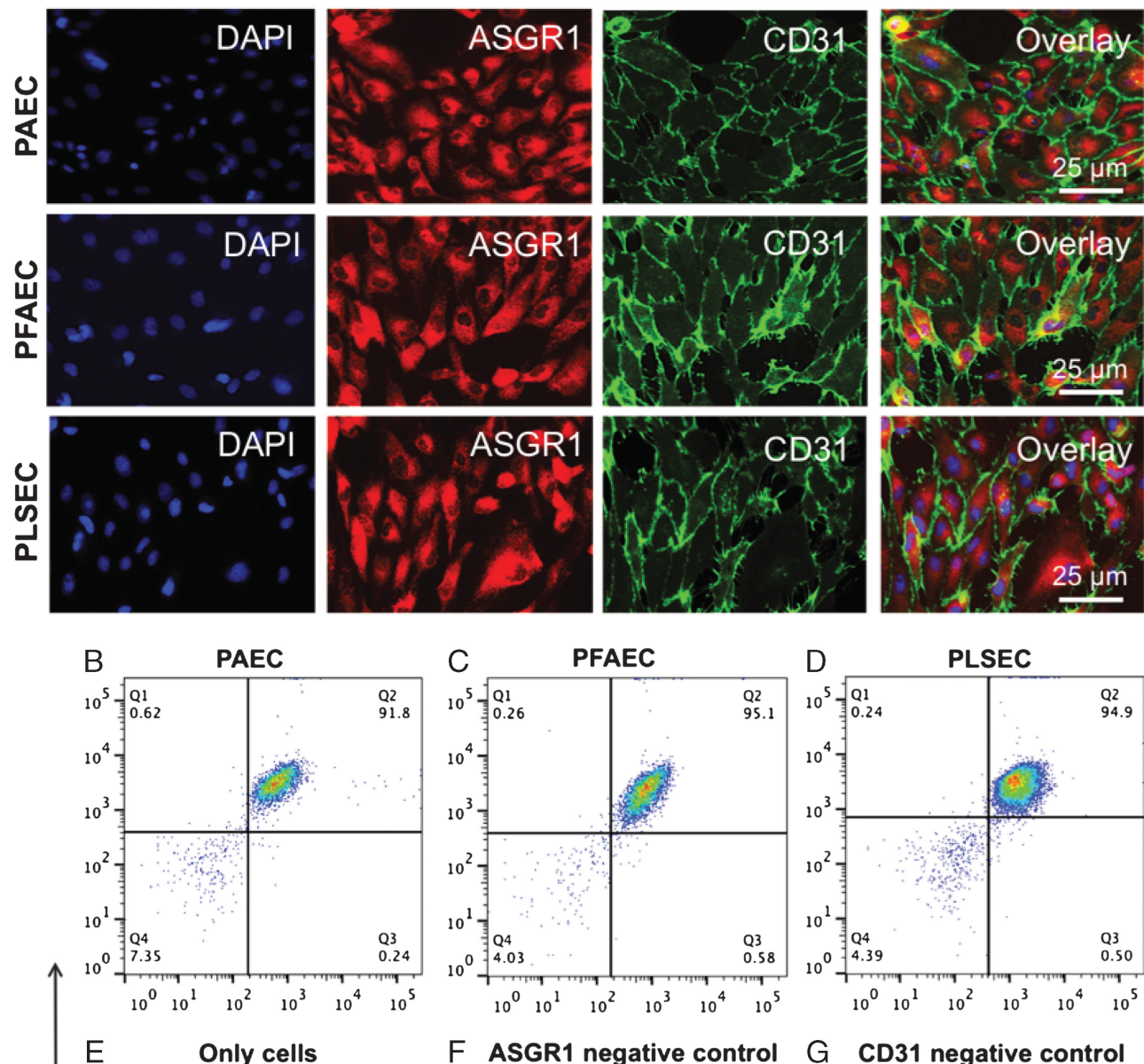

F ASGR1 negative control
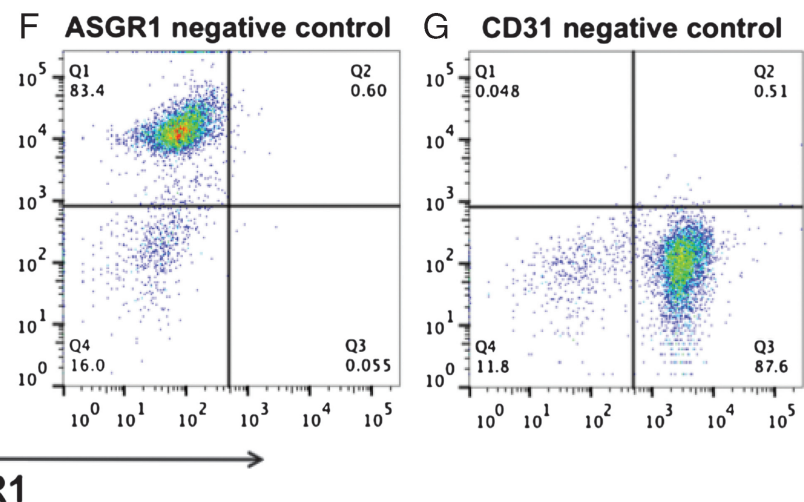

ASGR1

$\mathrm{H}$

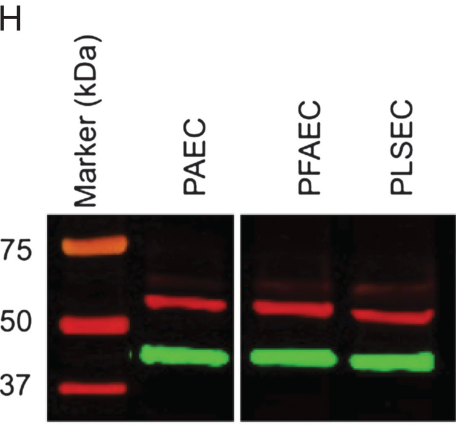

ASGR1 ( 50 kDa)

$\beta$-Actin (42 kDa)
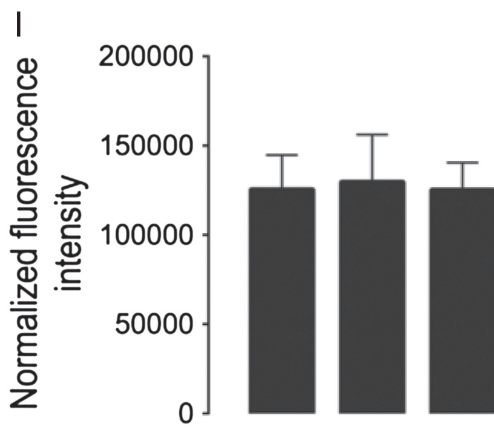

Copyright $@ 2015$ Wolters Kluwer Health, Inc. All rights reserved. 
arrows), indicating binding of platelet aggregates to the endothelium via ASGR1.

\section{Platelet Deposition on PAEC in a Microcarrier-Based in Vitro Xenotransplantation Model}

PAEC grown on microcarrier beads were incubated with fresh, non-anticoagulated human blood. The mean clotting time in the presence of PAEC was significantly prolonged as compared to microcarrier beads without PAEC (with PAEC, $40.3 \pm 8.1$ minutes vs $12.8 \pm 4.9$ minutes without PAEC; 2 -tailed $t$ test, $P=0.007)$. Blood-bead mixture was sampled after 10 minutes and 20 minutes of incubation and analyzed for platelet-lymphocyte, platelet-neutrophil, and plateletmonocyte staining by flow cytometry. Platelet counts dropped from $277.0 \pm 45.1 \times 10^{3} / \mu \mathrm{L}$ at baseline to $119.1 \pm 88.4 \times$ $10^{3} / \mu \mathrm{L}(P<0.01$ vs baseline $)$ after 10 minutes, and $35.0 \pm 43.0 \times 10^{3} / \mu \mathrm{L}(P<0.01$ vs baseline; $P<0.05$ vs 10 minutes) after 20 minutes of incubation. Baseline values for percentage of white blood cell (WBC)-platelet aggregates were $12.1 \% \pm 3.8 \%$ for lymphocytes, $6.7 \% \pm 3.3 \%$ for neutrophils, and $18.6 \% \pm 5.3 \%$ for monocytes. No significant changes in CD42a-positive platelet aggregation with lymphocytes $(15.9 \% \pm 14.6 \%$ and $16.5 \% \pm 3.9 \%)$ and with neutrophils $(14.0 \% \pm 7.3 \%$ and $19.3 \% \pm 3.3 \%)$ were observed after 10 and 20 minutes of incubation, respectively. In contrast, the percentages of platelet-monocyte aggregates within the CD14-positive monocyte population were significantly increased $(41.8 \% \pm 6.0 \%$ after 10 minutes, and $44.4 \% \pm$ $3.9 \%$ after 20 minutes, for both $P<0.0001$ vs baseline) (Table S1, SDC, http://links.lww.com/TP/B100). In addition, no statistically significant correlation between reduction in platelet counts and occurrence of platelet-lymphocyte $\left(\mathrm{r}^{2}=0.951, P=0.142\right)$, platelet-neutrophil $\left(\mathrm{r}^{2}=0.993\right.$, $P=0.053)$, or platelet-monocyte $\left(r^{2}=0.935, P=0.164\right)$ aggregates was observed.

Beads were sampled after 20 minutes of incubation and analyzed for platelet deposition on PAEC/microcarrier beads. Immunofluorescence staining of microcarrier beads/PAEC for the endothelial marker CD31 and for the platelet marker CD41 showed that CD41-positive platelet deposition was colocalized with CD31 on microcarrier beads, suggesting that after 20 minutes of incubation, human platelets adhered to PAEC (Figure S2, SDC, http://links.lww.com/TP/B100).

\section{Phagocytosis of Carboxyfluorescein Diacetate Succinimidyl Ester-Labeled Human Platelets by Porcine Vascular Endothelial Cells}

Freshly isolated human platelets were labeled with CFSE and incubated with cultured PAEC, PFAEC, and PLSEC in vitro. Within minutes, human platelets bound to all types of
EC, with a peak of fluorescence intensity at 15 minutes. There was nonstatistically significant difference in fluorescence intensity between different types of EC $(P \geq 0.056)$. From 30 to 60 minutes, the fluorescence declined to approximately $50 \%$ of the maximum (Figure $4 \mathrm{~A}$ ).

As shown by overlay immunofluorescence staining for CD31 (red), CFSE-labeled human platelets adhered to the surface of EC after 30 minutes of incubation (Figure 4B). After 45 minutes, however, CFSE-labeled human platelets were internalized by EC, and colocalization of fluorescence for ASGR1 and the lysosomal marker CD107a was observed (Figure 4C).

\section{Inhibition of Platelet Phagocytosis by Blocking of the ASGR1 Receptor}

To confirm involvement of ASGR1 in human platelet phagocytosis, we examined the effect of blocking the ASGR1 receptor. Preincubation of PAEC, PFAEC, and PLSEC with polyclonal rabbit anti-ASGR1 antibody led to a significant, dose-dependent reduction of platelet binding. At $2 \mu \mathrm{g} / \mathrm{mL}$, significance levels were $P<0.01, P<0.05$, and $P<0.01$ for PAEC, PFAEC, and PLSEC, respectively. At $5 \mu \mathrm{g} / \mathrm{mL}$, the significance of the reduction of platelet binding was $P<$ 0.0001 for all tested EC. Use of a nonspecific isotype control (polyclonal rabbit $\operatorname{IgG}$ ) had no effect on platelet binding (Figure 5A). In addition, asialofetuin, a glycoprotein lacking terminal sialic acid, was tested as a competitive ligand for ASGR1 in platelet binding assays. Also, for asialofetuin, a clear, dose-dependent reduction of platelet binding was observed, with $P<0.01$ to $<0.001$ for $2 \mu \mathrm{g} / \mathrm{mL}$ and $P<0.001$ to $<0.0001$ for $5 \mu \mathrm{g} / \mathrm{mL}$ (Figure $5 \mathrm{~B}$ ).

\section{DISCUSSION}

During ex vivo xenoperfusion of porcine limbs with heparinized human blood, human platelets disappeared within minutes from the circulating blood. This phenomenon is in line with the observation of xenograft-induced thrombocytopenia and represents a major problem in pig-to-primate xenotransplantation. ${ }^{8}$ Transplantation of organs from $\alpha 1,3$-galactosyltransferasegene-knockout (GalTKO) or GalTKO and human CD46 transgenic pigs also led to thrombocytopenia throughout the posttransplant period. ${ }^{11}$ Determination of the mechanisms responsible for this thrombocytopenia is necessary for the development of preventive strategies. In this study, we have shown the expression of ASGR1, a known platelet binding C-type lectin receptor, in PAEC as well as PFAEC. In addition to platelet activation and aggregation, we showed, by specific inhibition using polyclonal antiASGR1 antibody, that human platelet binding and uptake by PAEC and PFAEC is mediated via ASGR1.

FIGURE 2. Expression of ASGR1 on PAEC, PFAEC, and PLSEC in vitro. A, Cultured PAEC (upper row), PFAEC (middle row), or PLSEC (lower row) were analyzed for expression of ASGR1 as well as the endothelial specific marker CD31 by immunofluorescence staining and fluorescence microscopy. PAEC, PFAEC, and PLSEC were all positive for CD31 and ASGR1. Nuclei stained with DAPI. One representative of at least 3 independent experiments is shown. B, PAEC, C, PFAEC, and D, PLSEC were analyzed for expression of ASGR1 and CD31 by flow cytometry. One representative of 3 independent experiments is shown. E, Representative image of PAEC only, without any antibody staining. F, ASGR1/ CD31 staining on PAEC in the presence of both ASGR1 and CD31 primary antibodies and only the secondary antibody against anti-CD31. G, ASGR1/CD31 staining on PAEC in the presence of ASGR1 and C31 primary antibodies but only the secondary antibody against antiASGR1. These controls were used to test non-specific binding. $\mathrm{H}$, Western blot analysis of ASGR1 protein and $\beta$-actin from whole cell lysate using PAEC, PLAEC, and PLSEC cultures. Representative data of 3 independent experiments are shown. I, Quantitative analysis of Western blot bands of ASGR1/ß-actin from whole cell lysates of PAEC, PFAEC and PLSEC by ImageJ software. Shown are mean values \pm standard deviations of 3 high-power viewing fields each of at least 3 independent experiments. No significant differences in ASGR1 expression levels were observed as tested by 1-way ANOVA with Bonferroni correction. 
Asialoglycoprotein receptor-1, a component of the AshwellMorell asialoglycoprotein receptor, is located on hepatocytes, macrophages, and LSEC, and mediates binding and removal of circulating glycoproteins carrying Gal $\beta 1-4-$ or GalNAcß1-4-terminating epitopes. ${ }^{21,26}$ Asialoglycoprotein receptor-1 was previously considered a hepatocyte-specific protein, ${ }^{27}$ but has also been shown to occur extrahepatically in thyroid, ${ }^{28}$ small and large intestines, ${ }^{29,30}$, and testis. ${ }^{31}$ Its existence in renal proximal tubular epithelial cells has also been studied, ${ }^{32}$ whereas it has never been described to occur
A
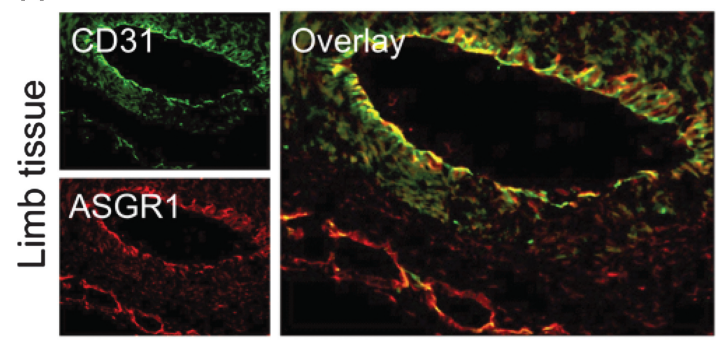

C

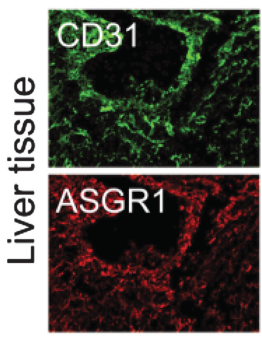

$\mathrm{E}$

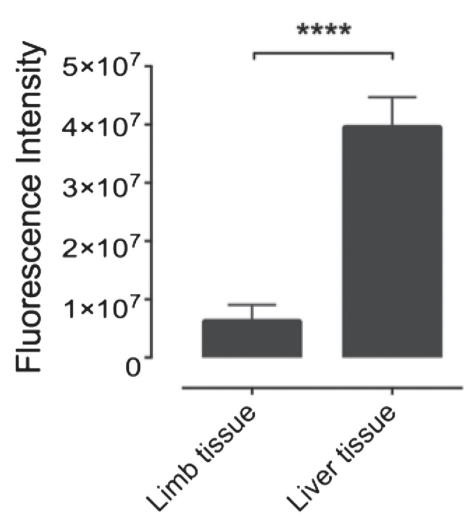

$\mathrm{H}$
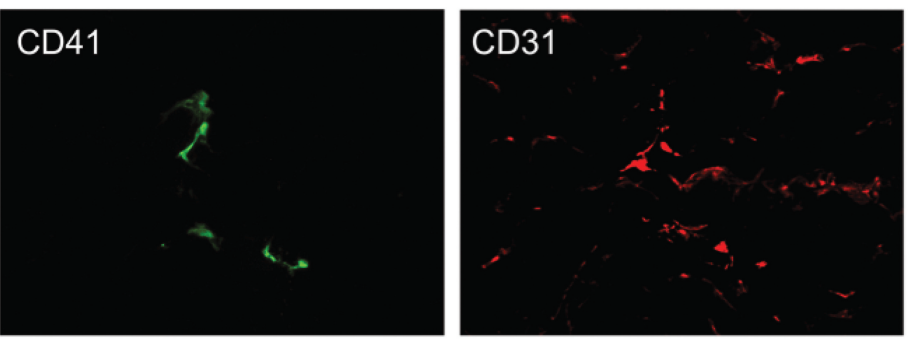

I
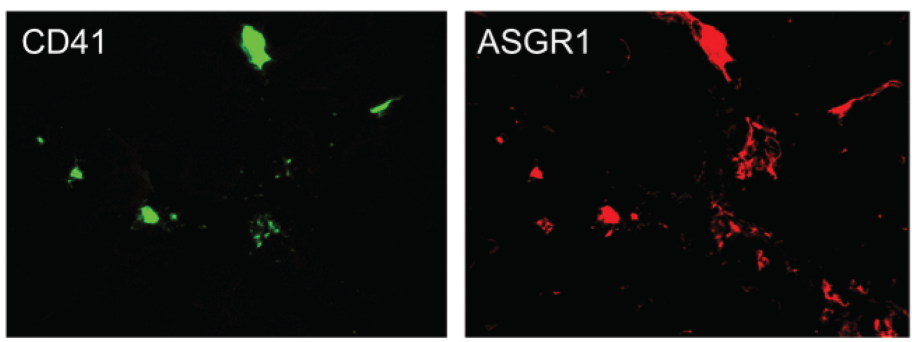

B
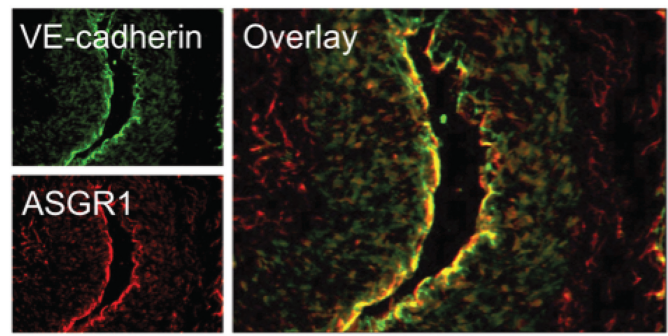

D
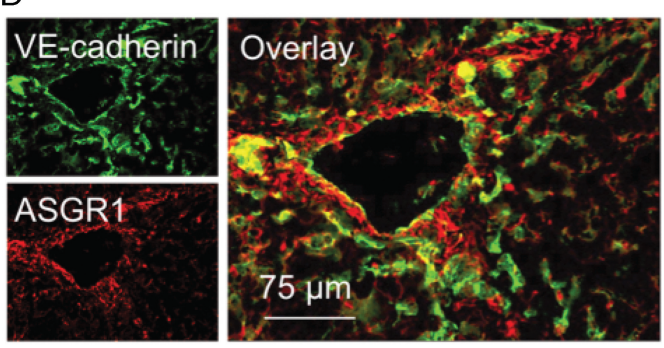

G
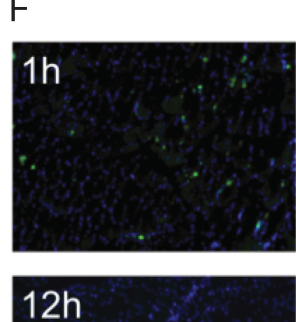

$250 \mu \mathrm{m}$

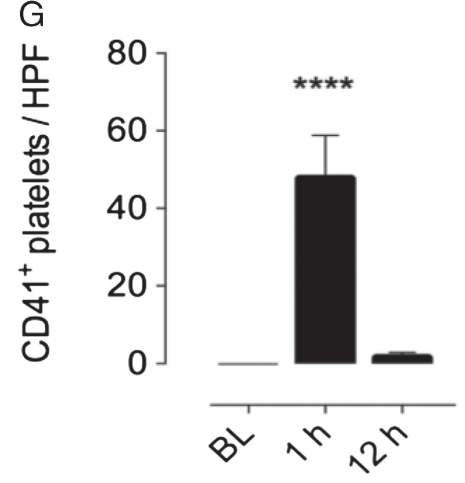

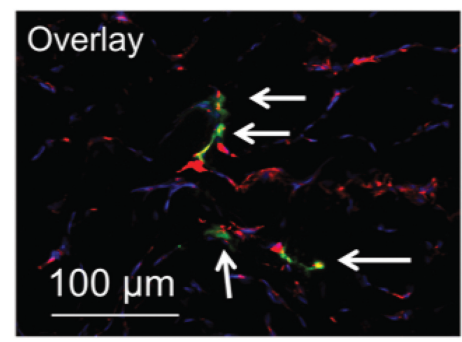

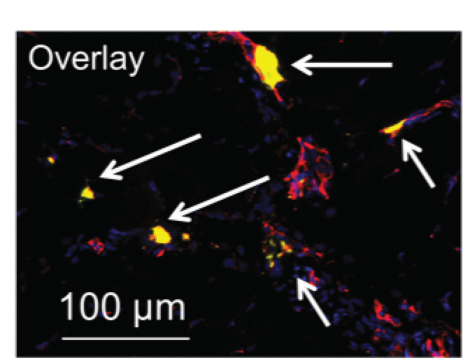



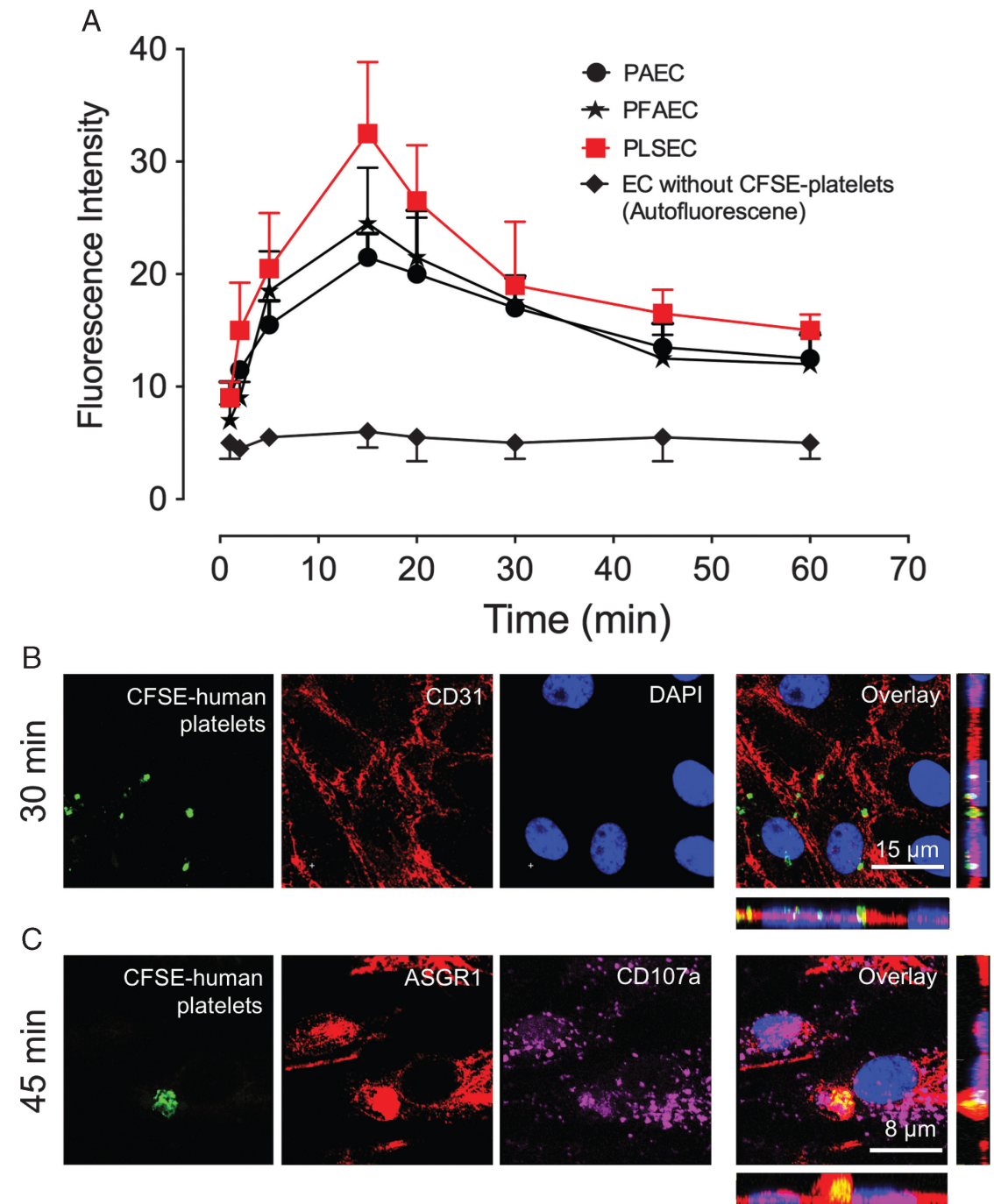

FIGURE 4. Human platelet phagocytosis and degradation by porcine vascular EC. (A) CFSE-labeled human platelets were incubated with porcine vascular EC for different time points as shown on the $x$ axis, followed by detection of CFSE fluorescence intensity on a plate reader. All ECs were also measured without CFSE-labeled platelets to determine the level of autofluorescence. Data are expressed as mean \pm SD of 3 independent experiments. (B) Confocal microscopy analysis of CFSE-labeled human platelets by PAEC in vitro. After 30 minutes of incubation, CFSElabeled platelets (green fluorescence) adhered to PAEC that were positive for CD31 (red fluorescence). Side views are shown as z panels to the right of and below the overlay image. (C) Prolonged incubation of CFSE platelets for 45 minutes resulted in internalization into PAEC. Shown are PAEC stained for ASGR1 (red) and the lysosomal marker CD107a (purple). Side views are shown as z panels to the right and below the overlay image. In all fluorescence staining experiments, nuclei were stained with DAPI. One representative of 3 independent experiments is shown.

on endothelial cells outside the liver. Our data now show that in addition to LSEC, extrahepatic vascular endothelial cells also express ASGR1. In vitro, porcine vascular (aortic and femoral arterial) endothelial cells, characterized by expression of the endothelial specific markers VE-cadherin and CD31, were shown to express ASGR1 by immunofluorescence staining, flow cytometry, and Western blot. Taken together, these assays demonstrate that the ASGR1 protein is present on PAEC as well as PFAEC.

The asialoglycoprotein receptor is composed of 2 subunits, a major subunit (ASGR1) and a minor subunit (ASGR2), 33,34 both of which are necessary to form a functional trimer of 2 ASGR1 and 1 ASGR2. ${ }^{35,36}$ Staining of limb muscle tissue sections confirmed the presence of ASGR1 in vascular

FIGURE 3. ASGR1 expression on biopsy samples from ex vivo perfusions. A and B, Pig limb skeletal muscle biopsies, collected before perfusion from the contralateral extremity as baseline samples, and (C and D) liver tissue samples were stained for expression of ASGR1 and endothelial markers (CD31 and VE-cadherin). Results show that ASGR1 protein is expressed in vascular endothelial cells that are positive for CD31 or VE-cadherin, as well as in hepatocytes. Representative data of 3 independent experiments are shown. E, Quantitative analysis of ASGR1 staining on limb and liver tissue samples by ImageJ software. Shown are mean values \pm standard deviations of 3 high-power viewing fields each of at least 3 independent experiments. Significance was tested using the unpaired 2-tailed Student $t$ test $\left.{ }^{(\star \star \star \star} P \leq 0.0001\right)$. F, Biopsy samples, collected at different xenoperfusion time points, were stained for platelets using anti-CD41 antibody to study binding and sequestration. G, CD41-positive human platelet binding and sequestration was high at 1 hour perfusion and then declined to baseline levels at 12 hours xenoperfusion. The amount of platelets was expressed as CD41+ platelets per HPF. Significance was tested by 1 -way ANOVA with Bonferroni correction $\left(^{\star \star \star \star} P \leq 0.0001\right)$. H and I, Staining for colocalization of human platelets with pig CD31 and/or ASGR1 on 1h xenoperfused pig limb biopsy samples. Human platelets stained with CD41 were colocalized with porcine (H) CD31 and (I) ASGR1. HPF, high-power viewing field. 

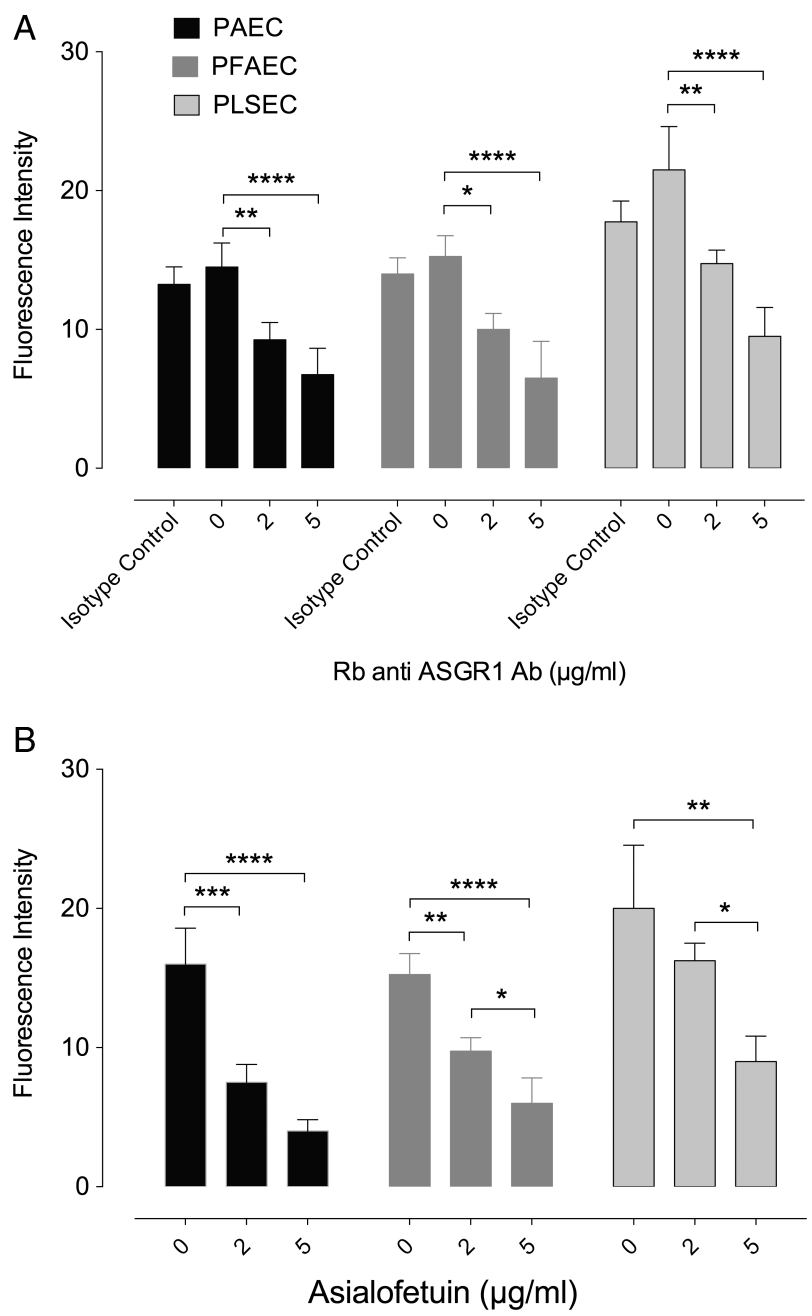

FIGURE 5. Inhibition of human platelet binding and phagocytosis. PAEC, PFAEC, and PLSEC were preincubated with increasing concentrations $(0-5 \mu \mathrm{g} / \mathrm{mL})$ of either $(A)$ rabbit polyclonal anti-ASGR1 antibody or polyclonal rabbit lgG as isotype control, or (B) asialofetuin, for 30 minutes, followed by incubation with CFSE-labeled human platelets. After 15 minutes of incubation, human platelet binding was measured using a fluorescence plate reader. Significance was tested using one-way ANOVA with Bonferroni correction $\left({ }^{\star} P \leq 0.05\right.$, $\left.{ }^{\star \star} P \leq 0.01,{ }^{* \star \star} P \leq 0.001,{ }^{* \star \star *} P \leq 0.0001\right)$. Data are expressed as mean \pm SD of 3 independent experiments.

endothelial cells. In addition to PLSEC, other liver cells including hepatocytes express ASGR1 and thus comparative analysis showed significantly higher ASGR1 staining on liver than limb tissue samples. Furthermore, muscle tissue biopsies from porcine limbs, ex vivo xenoperfused for 1 hour with human blood, stained positive for CD41, indicating human platelet sequestration. Staining for CD41 colocalized with ASGR1 protein, suggesting ASGR1-dependent human platelet binding and sequestration.

Platelet activation, aggregation, and phagocytosis can occur through multiple mechanisms in xenoperfusion or xenotransplantation. In addition, platelets have the potential to influence the immune responses through mechanisms involving endothelial cells and leukocytes. In a recent study, high amounts of platelet-platelet and platelet-WBC aggregates were found and linked to low apparent platelet counts. ${ }^{7,11}$ In the present study, we also observed plateletWBC aggregation after 10 and 20 minutes of incubation of whole, non-anticoagulated human blood with PAEC grown on microcarrier beads. These data suggest that platelet aggregation with WBC might be a common phenomenon in pigto-primate xenotransplantation or xenoperfusion. However, the reduction in platelet counts showed no statistically significant correlation with the percentage of platelet-WBC aggregation. Therefore, other mechanisms, particularly receptormediated adhesion to EC, may contribute to the reduction in platelet counts. This was supported by detection of $\mathrm{co}^{-}$ localized deposition of CD41-positive platelets with PAEC/ CD31 on microcarrier beads that are covered with a monolayer of PAEC, after incubating with human blood. Furthermore, our in vitro results using freshly isolated human platelets and porcine vascular EC suggest that, at least in part, receptor-ligand interactions are involved in the clearance of human platelets in vitro and ex vivo in pig-tohuman limb xenotransplantation models.

In vitro, binding of human platelets to EC was observed and peaked after 15 minutes incubation as reported previously using PLSEC and CFSE-labeled human platelets. ${ }^{20,21}$ Thereafter, the CFSE fluorescence elicited by the labeled platelets gradually declined and at 45 minutes was colocalized with CD107a, a marker for lysosomes. These findings are in line with the finding that the receptor forms a complex with galactose-terminated glycoproteins, which are internalized and transported to lysosomes, as indicated by colocalized deposition of CFSE platelets, ASGR1, and CD107a. The receptor and ligand dissociate, and the receptor is recycled back to the cell surface. ${ }^{37}$

Asialoglycoprotein receptor-1 mediates endocytosis of glycoproteins from which the terminal sialic acid residues have been removed. The presence of higher Gal $\beta 1-4$ or GalNAc $\beta 1-4$ ligand concentrations on human as compared to porcine platelets may facilitate porcine ASGR1-mediated human platelet phagocytosis. ${ }^{21}$ Platelet aggregation and phagocytosis by PLSEC has also been reported in pig-to-baboon liver xenotransplantation and can be prevented by blocking integrin adhesion pathways. ${ }^{38}$ Indeed, preincubation of PAEC with polyclonal anti-ASGR1 antibody significantly reduced human platelet binding. Also, blocking of ASGR1 using asialofetuin, a competitive ligand for ASGR1, significantly reduced platelet uptake by EC in a dose-dependent manner, supporting involvement of the porcine ASGR1 in human platelet phagocytosis. Generation of ASGR1 knockout pigs, with or without introduction of the human ASGR1 and on top of GalTKO and expression of human regulatory proteins for complement and coagulation, may therefore help to overcome the problem of thrombocytopenia in pig-to-human xenotransplantation.

In conclusion, we found expression of functional ASGR1 in extrahepatic porcine vascular endothelial cells. ASGR1 on the vascular endothelium of porcine organs may therefore mediate binding and phagocytosis of human platelets during pig-to-human xenotransplantation.

\section{ACKNOWLEDGMENTS}

The authors thank Dr. Daniel Mettler, Mrs. Olgica Beslac and Mr. Daniel Zalokar from the Experimental Surgery Unit, as well as Pavan Garimella, Alain Despont, Yvonne Roschi, and Sanja Stojanovic, Department of Clinical Research, University of Bern, and Dr. Amiq Gazdhar from the Division 
of Pulmonary Medicine, Bern University Hospital, for expert technical support. The authors also thank PD Dr. Beat Schnüriger from the Clinic of Visceral Surgery, Bern University Hospital, for support with pig liver excision and cannulation. Images were acquired on equipment and service supported by the Microscopy Imaging Center of the University of Bern.

\section{REFERENCES}

1. Cozzi E, White DJ. The generation of transgenic pigs as potential organ donors for humans. Nat Med. 1995;1:964.

2. McCurry KR, Kooyman DL, Alvarado CG, et al. Human complement regulatory proteins protect swine-to-primate cardiac xenografts from humoral injury. Nat Med. 1995;1:423.

3. Chen D, Weber M, McVey JH, et al. Complete inhibition of acute humora rejection using regulated expression of membrane-tethered anticoagulants on xenograft endothelium. Am J Transplant. 2004;4:1958.

4. Kuwaki K, Tseng YL, Dor FJ, et al. Heart transplantation in baboons using alpha1,3-galactosyltransferase gene-knockout pigs as donors: initial experience. Nat Med. 2005;11:29.

5. Shimizu A, Hisashi $Y$, Kuwaki K, et al. Thrombotic microangiopathy associated with humoral rejection of cardiac xenografts from alpha1,3galactosyltransferase gene-knockout pigs in baboons. Am J Pathol. 2008:172:1471

6. Ekser B, Ezzelarab M, Hara H, et al. Clinical xenotransplantation: the next medical revolution? Lancet. 2012;379:672.

7. Iwase $\mathrm{H}$, Ekser $\mathrm{B}$, Zhou $\mathrm{H}$, et al. Platelet aggregation in humans and nonhuman primates: relevance to xenotransplantation. Xenotransplantation. 2012;19:233.

8. Ekser B, Long C, Echeverri GJ, Dons EM, Cooper DK, Ezzelarab MB. Impact of thrombocytopenia on survival of baboons with genetically modified pig liver transplants: clinical relevance. Am J Transplant. 2010;10:273.

9. Hickman R, Parker JR, Saunders SJ, Terblanche J. Platelet adherence in the pig liver perfused with human blood. Transplantation. 1972;13:195.

10. Ekser B, Burlak C, Waldman JP, et al. Immunobiology of liver xenotransplantation. Expert Rev Clin Immunol. 2012;8:621.

11. Ezzelarab M, Ekser B, Gridelli B, Iwase H, Ayares D, Cooper DK. Thrombocytopenia after pig-to-baboon liver xenotransplantation: where do platelets go? Xenotransplantation. 2011;18:320.

12. Burlak $C$, Paris $L L$, Chihara RK, et al. The fate of human platelets perfused through the pig liver: implications for xenotransplantation. Xenotransplantation. 2010;17:350.

13. Nedredal GI, Elvevold KH, Ytrebo LM, Olsen R, Revhaug A, Smedsrod B. Liver sinusoidal endothelial cells represents an important blood clearance system in pigs. Comp Hepatol. 2003;2:1.

14. Stockert RJ. The asialoglycoprotein receptor: relationships between structure, function, and expression. Physiol Rev. 1995;75:591.

15. Ashwell G, Kawasaki T. A protein from mammalian liver that specifically binds galactose-terminated glycoproteins. Methods Enzymol. 1978; 50:287.

16. Hildenbrandt GR, Aronson NN Jr. Uptake of asialoglycophorin-liposomes by the perfused rat liver. Biochim Biophys Acta. 1980;631:499.

17. Dini L, Autuori F, Lentini A, Oliverio S, Piacentini M. The clearance of apoptotic cells in the liver is mediated by the asialoglycoprotein receptor. FEBS Lett. 1992;296:174.

18. Weigel PH. Galactosyl and N-acetylgalactosaminyl homeostasis: a function for mammalian asialoglycoprotein receptors. Bioessays. 1994;16:519.
19. Kolb-Bachofen V, Schlepper-Schafer J, Vogell W, Kolb H. Electron microscopic evidence for an asialoglycoprotein receptor on Kupffer cells: localization of lectin-mediated endocytosis. Cell. 1982;29:859.

20. Paris LL, Chihara RK, Reyes LM, et al. ASGR1 expressed by porcine enriched liver sinusoidal endothelial cells mediates human platelet phagocytosis in vitro. Xenotransplantation. 2011;18:245.

21. Paris LL, Chihara RK, Sidner RA, Tector AJ, Burlak C. Differences in human and porcine platelet oligosaccharides may influence phagocytosis by liver sinusoidal cells in vitro. Xenotransplantation. 2012;19:31.

22. Bongoni AK, Kiermeir D, Jenni H, et al. Complement dependent early immunological responses during ex vivo xenoperfusion of hCD46/HLA-E double transgenic pig forelimbs with human blood. Xenotransplantation. 2014;21:230.

23. Banz $Y$, Cung $T$, Korchagina $E Y$, et al. Endothelial cell protection and complement inhibition in xenotransplantation: a novel in vitro model using whole blood. Xenotransplantation. 2005;12:434.

24. Kohler HP, Muller M, Bombeli T, et al. The suppression of the coagulation of nonanticoagulated whole blood in vitro by human umbilical endothelial cells cultivated on microcarriers is not dependent on protein $\mathrm{C}$ activation. Thromb Haemost. 1995;73:719.

25. Wuensch A, Baehr A, Bongoni AK, et al. Regulatory sequences of the porcine THBD gene facilitate endothelial-specific expression of bioactive human thrombomodulin in single- and multitransgenic pigs. Transplantation. 2014:97:138

26. Harris RL, van den Berg CW, Bowen DJ. ASGR1 and ASGR2, the Genes that Encode the Asialoglycoprotein Receptor (Ashwell Receptor), are expressed in peripheral blood monocytes and show interindividual differences in transcript profile. Mol Biol Int. 2012;2012:283974.

27. Weigel PH. Characterization of the asialoglycoprotein receptor on isolated rat hepatocytes. J Biol Chem. 1980;255:6111.

28. Pacifico F, Laviola $L$, Ulianich $L$, et al. Differential expression of the asialoglycoprotein receptor in discrete brain areas, in kidney and thyroid. Biochem Biophys Res Commun. 1995;210:138.

29. Mu JZ, Fallon RJ, Swanson PE, Carroll SB, Danaher M, Alpers DH. Expression of an endogenous asialoglycoprotein receptor in a human intestinal epithelial cell line, Caco-2. Biochim Biophys Acta. 1994;1222:483.

30. Mu JZ, Gordon M, Shao JS, Alpers DH. Apical expression of functional asialoglycoprotein receptor in the human intestinal cell line HT-29. Gastroenterology. 1997;113:1501.

31. Abdullah M, Kierszenbaum AL. Identification of rat testis galactosyl receptor using antibodies to liver asialoglycoprotein receptor: purification and localization on surfaces of spermatogenic cells and sperm. J Cell Biol. 1989;108:367.

32. Seow YY, Tan MG, Woo KT. Expression of a functional asialoglycoprotein receptor in human renal proximal tubular epithelial cells. Nephron. 2002;91:431.

33. Halberg DF, Wager RE, Farrell DC, et al. Major and minor forms of the rat liver asialoglycoprotein receptor are independent galactose-binding proteins. Primary structure and glycosylation heterogeneity of minor receptor forms. J Biol Chem. 1987:262:9828.

34. Bischoff J, Lodish HF. Two asialoglycoprotein receptor polypeptides in human hepatoma cells. J Biol Chem. 1987;262:11825.

35. Grewal PK, Uchiyama S, Ditto D, et al. The Ashwell receptor mitigates the lethal coagulopathy of sepsis. Nat Med. 2008;14:648.

36. Tozawa R, Ishibashi S, Osuga J, et al. Asialoglycoprotein receptor deficiency in mice lacking the major receptor subunit. Its obligate requirement for the stable expression of oligomeric receptor. J Biol Chem. 2001;276:12624.

37. Yik JH, Weigel PH. The position of cysteine relative to the transmembrane domain is critical for palmitoylation of $\mathrm{H} 1$, the major subunit of the human asialoglycoprotein receptor. J Biol Chem. 2002;277:47305

38. Peng QYH, Enjoji K, Machaidze Z, Wei L, et al. Integrin-dependent baboon platelet activation and phagocytosis by porcine hepatocytes and endothelial cells. Xenotransplantation. 2011;18:287. 